\title{
GROWTH AND PHYSIOLOGICAL ATTRIBUTES OF SOYBEAN SEEDLING (Glycine max L.) UNDER SALINITY STRESS
}

El-Rodeny, W. M. ${ }^{1}$; Samira A. F. H. EL-Okkiah ${ }^{2}$; A. R. Morsy ${ }^{3}$; E.A. elghazaly ${ }^{4}$ and soad El-Sayed ${ }^{5}$

1, 3, 4 Food Legumes Res. Sec. Field Crops Res. Inst., Agric. Res., Center (ARC)

2 Agricultural Botany Dept., Fac. Agric.-Kafr El-Sheikh University.

5 Seed Technology Res. Dep., Field Crops Res. Inst., Agric. Res., Center (ARC),, Egypt.

wrodeny@yahoo.com

\begin{abstract}
The present study was carried out at Sakha Agric. Research Station during 2011 growing season to study the germination and early seedling growth and physiological attributes of five soybean (Glycine max L. Merr.) cultivars (Toano, Giza 35, Giza 111, Giza 22 and Giza 83, beside the five breeding lines H32, DR. 101, H9L186, H6L136 and H2L78) under different levels of saline water. Two experiments were conducted first experiment on Petri dishes and the other in pots and saline water concentration, were $0,6 \mathrm{ds} / \mathrm{m} 9 \mathrm{ds} / \mathrm{m}, 12 \mathrm{ds} / \mathrm{m}$ and $15 \mathrm{ds} / \mathrm{m}$. The obtained results proved that the genotypes under study could be arranged into three groups according to their sensitivity to salinity stress. The first group included the genotypes DR 101 , Toano, H2L78, H9L86 and Giza 83, which are the most tolerant to salinity due to the high germinability and vigorous growth to development for whole plant under all levels of salinity. The second group included H32 and Giza 111 which are moderate tolerant. The third group include; H6L136, Giza 22 and Giza 35 genotypes as susceptible genotypes under different levels of salinity. Determination of ions showed that $\mathrm{Na}+$ concentration increased with increasing salinity level in all of genotypes. While the $\mathrm{K}+$ concentration was higher in salt stress tolerant soybean plants than the susceptible ones which decreased $\mathrm{Na}+$ toxicity. It could be concluded that soybean genotypes Toano, Giza 83 and DR. 101 are salt stress tolerant due to their less $\mathrm{Na}+$ absorption and more $\mathrm{Na}+$ accumulation in root compared with other studied genotypes. The results indicated that under salt stress plant growth of cultivars Toano, Giza 83 and DR. 101 were better compared to the other soybean genotypes.
\end{abstract}

Keywords: Soybean, (Glycine max L.), genetic evaluation, salinity, germination, nutrient Content.

\section{INTRODUCTION}

Soil and water salinity is recognized as the most important problem involved in crop plants establishment and growth. Transpiration and evaporation from the soil surface, low quality of irrigation water and lack of proper drainage are major causes of soil salinity leading to crop loss. Area of World salty land is thought to be 950 million hectares. Thus, screening of salt tolerant crop cultivars is of crucial importance (Okçu et al. 2005). Soybean is a member of family Fabaceae and the world foremost provider of protein and oil. It is often called the miracle crop as it contains high protein content (38$45 \%)$ as well as high oil content (20\%). High sensitivity to soil and water salinity is one of the biggest problems with soybean crop. Reports have indicated that salinity affects growth and development of plants through 
osmotic and ionic stresses. Because of accumulated salts in soil under salt stress condition plant wilts apparently while soil salts such as $\mathrm{Na}+$ and $\mathrm{Cl}$ disrupt normal growth and development of plant (Khajeh-Hosseini et al. 2003.) (Chen et al. 1996) reported that salt stress led to decreased seedling growth of soybean cultivars. Rogeress et al. 1994 suggested that salt stress decreased germination percentage of white clover. Cultivars accumulating $\mathrm{Na}$ + and $\mathrm{Cl}$-in roots and inhibited transportation of these ions to air organs were more resistant to salt stress circumstances. Salinity led to decreased dry weight of shoot and root. (Farhoudi et al. 2007) found that salt tolerance of canola cultivars have a direct relationship with $\mathrm{Na}+/ \mathrm{K}+$ ratio so that the ratio increased with the increase of salinity level but less increase is observed in tolerant cultivars, They concluded that $\mathrm{Na}+/ \mathrm{K}+$ ratio can be a measure of salt stress tolerance. (Farhoudi and Tafti 2011) indicated that salt stress led to decrease germination, seedling fresh weight, seedling growth and seedling $\mathrm{K}+$ percentage in dry matter but increase mean germination time and seedling $\mathrm{Na}$ +percentage. Therefore the main objective of the present work is study the germination and early seedling growth and physiological attributes of some soybean (Glycine max L. Merr.) genotypes under different levels of saline water.

\section{MATERIALS AND METHODS}

\section{Plant material and growth conditions}

Ten soybean genotypes with different background obtained from Food Legumes Research Section at Sakha Agric. Research Station (SARS). The studied cultivars were H32, (Giza 21(Crawford x Celeste) X L86 -K-73) Toano, Giza 35, DR. 101, Giza 111, Giza 22 and Giza 83, besides the breeding lines H9L186 (Toano x Kent), H6L136 (Toano x AGS 129) and H2L78 (DR.101x Giza111). These cultivars represent different maturity groups according to the U.S. Classification, i.e. Giza 83(Maturity Group II), H32 and Giza 35 (MG III), Giza 22, Giza 111 and H2L78 (MG IV), DR. 101, H9L186, H6L136 and Toano (MG V). Seeds of these cultivars and promising lines were sown using a randomized complete blocks design with three replications. All genotypes were irrigated by five levels of saline water, 0, 6 $\mathrm{ds} / \mathrm{m}, 9 \mathrm{ds} / \mathrm{m} 12 \mathrm{ds} / \mathrm{m}$ and $15 \mathrm{ds} / \mathrm{m}$.

Two experiments were conducted, first on Petri dishes; three replicates of 20 seeds of each cultivar were germinated in 2 rolled Whatman filter papers with $10 \mathrm{ml}$ of respective test solutions. The papers were replaced every 2 days to prevent accumulation of salts (Rehman et al., 1996). In order to prevent evaporation, each rolled paper was put into Petri dishes of $12 \mathrm{~cm}$ diameter and $2 \mathrm{~cm}$ depth. Seeds were allowed to germinate at $20 \pm 1^{\circ} \mathrm{C}$ in the dark for 10 days, to determine the toxic effects of the solutions on germination. A seed was considered to have germinated when the emerging radicle elongated to $1 \mathrm{~mm}$. Germination percentage was recorded every $24 \mathrm{~h}$ for 10 days. Radicle and shoot lengths were measured on the 10th day. The second, experiment in pots was executed on the same certain soybean genotypes were grown under five concentrations of $\mathrm{NaCl},(0,6,9,12,15 \mathrm{dS}$ $\mathrm{m}-1$ ) during 2011 summer season. Soybean genotypes were grown in plastic 
pots $30 \mathrm{~cm}$ in width and $30 \mathrm{~cm}$ in deep filled with $10 \mathrm{~kg}$ of disturbed non saline clay soil collected from the surface layer $(0-30 \mathrm{~cm})$ of SARS Farm. The chemical and physical properties of experimental soil are shown in Table 1.

Table (1): Some chemical and physical properties of soil used

\begin{tabular}{|c|c|c|c|c|c|c|c|c|c|c|c|}
\hline \multirow[b]{2}{*}{ Season } & \multirow[b]{2}{*}{$\begin{array}{c}\text { pH } \\
1: 2.5\end{array}$} & \multirow{2}{*}{$\begin{array}{l}\text { **ECe } \\
\text { dS/m }\end{array}$} & \multicolumn{4}{|c|}{ Soluble Cation, meq/L } & \multicolumn{4}{|c|}{ Soluble Anion, meq/L } & \multirow[b]{2}{*}{ SAR } \\
\hline & & & $\mathrm{Ca}^{++}$ & $\mathbf{M g}^{++}$ & $\mathrm{Na}^{+}$ & $\mathbf{K}^{+}$ & $\mathrm{CO}_{3}^{--}$ & $\mathrm{HCO}_{3-}$ & $\mathrm{Cl}^{-}$ & $\mathrm{SO}_{4--}$ & \\
\hline 2011 & 8.05 & 2.00 & 7.5 & 2.3 & 9.9 & 0.3 & - & 2.3 & 9.2 & 8.5 & 4.48 \\
\hline
\end{tabular}

Table 1 Cont.

\begin{tabular}{|l|c|c|c|c|c|c|c|c|c|}
\hline \multirow{2}{*}{ Season } & \multirow{2}{*}{$\begin{array}{c}\text { Total } \\
\text { N\% }\end{array}$} & \multicolumn{2}{|c|}{$\begin{array}{c}\text { Available } \\
\text { ppm }\end{array}$} & F.C. & O.M & \multicolumn{2}{|c|}{$\begin{array}{c}\text { Particle size } \\
\text { distribution }\end{array}$} & \multirow{2}{*}{ Text ure } \\
\cline { 3 - 9 } & & $\mathbf{P}$ & $\mathbf{K}$ & $\%$ & $\%$ & Clay & Silt & sand & \\
\hline 2011 & 1.12 & 6 & 320 & 40 & 1.6 & 55 & 23 & 22.6 & Clayey \\
\hline
\end{tabular}

* 1:2.5 Soil: Water suspension ${ }^{\star \star}$ Soil paste extract

Soybean genotypes were planted on 15th May, 2011. After two weeks of sowing the seedlings were thinned to two plants/pot. The first irrigation for each pot was done with saline water. After germination, constant volume of artificially sterilized water equivalent to field capacity was used for irrigation. Five levels of saline water were used for irrigation. The artificially saline water were prepared using a base of tap water with $\mathrm{Na}$ and $\mathrm{Ca}$ at SAR $=6$ by using a mixture of $\mathrm{CaCl}_{2}$ and $\mathrm{NaCl}$ Salts.

\section{Assessed traits:}

Germination percentage, seedling growth traits (radicle and shoot length,) plant growth traits (root length and plant height) and physiological traits $\left(\mathrm{Na}^{+}\right.$and $\mathrm{K}^{+}$contents of the plant shoot and root).

\section{$\mathrm{Na}^{+}$and, $\mathrm{K}+$ determination:}

For the determination of $\mathrm{Na}^{+}$and $\mathrm{K}^{+}$in the seedling, one mg dry mater was placed in test tubes containing $20 \mathrm{ml}$ distilled deionized water, and heated in a boiling water bath for $1 \mathrm{~h}$. The tubes were then autoclaved at $120^{\circ} \mathrm{C}$ for $20 \mathrm{~min}$ and cooled. The $\mathrm{Na}^{+}$and $\mathrm{K}^{+}$content in 15 time's diluted extract was determined by atomic absorption spectrophotometry (Ansari and Flowers, 1986)

The statistical analysis:

Data were analyzed using SPSS version 13 computer software package to test for significant effect of the treatments. Comparison of treatments was done by the least significant difference (LSD) at $5 \%$.

\section{RESULTS AND DISCUSSION}

The data in Table (2) showed that Increasing salt stress level, decreased soybean germination percentage. Under the highest salinity level, DR. 101 genotype had more germination percentage (90\%) compared to the other soybean genotypes. (Chippa and Rana 1995) reported that irrigation water with EC $14 \mathrm{ds} / \mathrm{m}$ significantly decreased the seed germination level of soybean. Results also showed that radicle and shoot length were significantly decreased with the increase of salinity level. The longest radicle and shoot were observed in the control treatment for toano and H9L186. Increase of 
salinity level to $12 \mathrm{dS} / \mathrm{m}$ and $15 \mathrm{dS} / \mathrm{m}$ treatments significantly decreased radicle and shoot length for all soybean genotypes. Radicle and shoot length of toano and Giza 111 were significantly highest among all genotypes under in $12 \mathrm{dS} / \mathrm{m}$ treatment. But no significant differences in shoot length were observed among all soybean genotypes under $15 \mathrm{dS} / \mathrm{m}$ treatment. These finding are in agreement with those obtained by Wang and Shannon 1999; who mentioned that the chloride salinity has been shown to affect the rate of germination, nodulation, leaflet size, shoot height, root length, shoot and root dry weight, and seed size and weight of soybeans.

The data in Table (3) showed that increase salinity level decreased plant height of studied soybean genotypes at 50 days from sowing. The highest plant height was obtained in the control treatment and 6 $\mathrm{dS} / \mathrm{m}$ while the lowest one was observed in $15 \mathrm{dS} / \mathrm{m}$ treatment. Similar results were achieved when root length was measured. The highest and the lowest root length were related to control treatment and $15 \mathrm{dS} / \mathrm{m}$ treatment, respectively. Researchers reported that accumulation of ions in plant growth environment causes osmotic and Pseudo-drought stress leading to a decrease of water absorption by plant tissues. Consequently the decrease of tissue water content results in reduction of cellular growth and development. Therefore, restriction of water absorption and its consequences for cellular growth and development is one of the most important causes of decreased growth of stem and root. Root cells have a much less turgor threshold pressure than that of stem cells thus root growth is more than stem growth under salt and drought stresses. Therefore, root is significantly less affected by salt stress than stem (Sadeghi, 2009 and Rahimi and Biglarifard, 2011).

In conclusion, the genotypes could be arranged into three groups depending on the means values. The first group included the genotypes DR101, Toano, H2L78, H9L86 and Giza 83, which are considered to be the most tolerant group to salinity with high ability for germination and to development for whole plant under five levels of salinity. The second group included H32 and Giza 111 which is moderate tolerant. The third group included; H6L136, Giza 22 and Giza 35 as susceptible genotypes accompanied with adverse effects in most traits under the different levels of salinity.

Salinity stress companied by increasing soil ions concentration and charging the ions balance decreased the germination and plant growth rate. The results of this investigation showed that salinity decreased the germination percentage, seedling fresh weight, shoot and root length of soybean genotypes. Results showed also that salt stress has increased soybean shoot $\mathrm{Na}^{+}$content but decreased $\mathrm{K}^{+}$content. $\mathrm{Na}^{+}$content of shoot tissues of the genotypes Toano, Giza 83 and DR. 101, was significantly lower than that of other genotypes in all treatments.). 
Table (2): Effect of salt stress on germination and seedling growth of some soybean genotypes

\begin{tabular}{|c|c|c|c|c|}
\hline \begin{tabular}{|l} 
Genotypes \\
H6L136
\end{tabular} & & Germination \% & $\frac{\text { Shoot length }}{6.62}$ & $\frac{\text { Radicle length }(\mathrm{cm})}{552}$ \\
\hline \begin{tabular}{|l}
$\mathrm{H} 6 \mathrm{~L} 136$ \\
$\mathrm{H} 9 \mathrm{~L} 186$
\end{tabular} & & $\begin{array}{l}59.02 \\
72.00\end{array}$ & $\begin{array}{l}6.62 \\
6.78\end{array}$ & $\begin{array}{l}5.52 \\
7.90\end{array}$ \\
\hline Toano & & 80.60 & 7.26 & 8.22 \\
\hline Hᄀ2L78 & & 68.00 & 5.36 & 4.93 \\
\hline Giza 35 & & 42.94 & 5.00 & 3.78 \\
\hline H32 & & 69.68 & 8.48 & 7.50 \\
\hline Giza 83 & & 72.34 & 8.74 & 7.08 \\
\hline DR. 101 & & 92.02 & 7.33 & 8.14 \\
\hline Giza 111 & & 73.32 & 7.92 & 8.08 \\
\hline Giza 22 & & 50.66 & 6.56 & 4.90 \\
\hline L.S.D. (0.05) & & 0.07 & 0.06 & 0.08 \\
\hline Salinity levels & & & & \\
\hline Control $(0)$ & & 73.32 & 10.99 & 8.68 \\
\hline $6 \mathrm{dS} / \mathrm{m}$ & & 70.31 & 7.97 & 6.97 \\
\hline $9 \mathrm{dS} / \mathrm{m}$ & & 69.68 & 7.07 & 6.57 \\
\hline $12 \mathrm{dS} / \mathrm{m}$ & & 68.65 & 5.12 & 6.03 \\
\hline $15 \mathrm{dS} / \mathrm{m}$ & & 58.33 & 3.87 & 4.77 \\
\hline L.S.D. (0.05) & & 0.049 & 0.049 & 0.048 \\
\hline & & & & \\
\hline H6L136 & Control (0) & 56.7 & 11.9 & 8 \\
\hline & $6 \mathrm{dS} / \mathrm{m}$ & 60 & 5.7 & 5.5 \\
\hline & $9 \mathrm{dS} / \mathrm{m}$ & 66.7 & 7.3 & 5.5 \\
\hline & $12 \mathrm{dS} / \mathrm{m}$ & 60 & 4.6 & 4.5 \\
\hline & $15 \mathrm{dS} / \mathrm{m}$ & 51.7 & 3.6 & 4.1 \\
\hline H9L186 & Control (0) & 71.7 & 13.5 & 12.1 \\
\hline & $6 \mathrm{dS} / \mathrm{m}$ & 73.3 & 6.1 & 7.4 \\
\hline & $9 \mathrm{dS} / \mathrm{m}$ & 81.7 & 6.2 & 8.4 \\
\hline & $12 \mathrm{dS} / \mathrm{m}$ & 75 & 4.6 & 7.2 \\
\hline & $15 \mathrm{dS} / \mathrm{m}$ & 58.3 & 3.5 & 4.4 \\
\hline Toano & Control (0) & 90 & 12.4 & 12.3 \\
\hline & $6 \mathrm{dS} / \mathrm{m}$ & 93.3 & 6.5 & 6.6 \\
\hline & $9 \mathrm{dS} / \mathrm{m}$ & 86.7 & 8 & 9.3 \\
\hline & $12 \mathrm{dS} / \mathrm{m}$ & 79.7 & 5.4 & 7.8 \\
\hline & $15 \mathrm{dS} / \mathrm{m}$ & 53.3 & 4 & 5.1 \\
\hline Hᄀ2L78 & Control (0) & 71.7 & 10.7 & 7.8 \\
\hline & $6 \mathrm{dS} / \mathrm{m}$ & 85 & 4.8 & 5.1 \\
\hline & $9 \mathrm{dS} / \mathrm{m}$ & 68.3 & 4.1 & 3.4 \\
\hline & $12 \mathrm{dS} / \mathrm{m}$ & 75 & 4.3 & 5 \\
\hline & $15 \mathrm{dS} / \mathrm{m}$ & 40 & 2.9 & 3.6 \\
\hline Giza 35 & Control (0) & 51.7 & 6.9 & 5.6 \\
\hline & $6 \mathrm{dS} / \mathrm{m}$ & 50 & 4 & 3 \\
\hline & $9 \mathrm{dS} / \mathrm{m}$ & 28.3 & 5.3 & 2.7 \\
\hline & $12 \mathrm{dS} / \mathrm{m}$ & 41.7 & 4.9 & 4.3 \\
\hline & $15 \mathrm{dS} / \mathrm{m}$ & 43.3 & 3.9 & 3.3 \\
\hline $\mathrm{H} 32$ & Control (0) & 65.0 & 11.4 & 8.5 \\
\hline & $6 \mathrm{dS} / \mathrm{m}$ & 76.7 & 12.4 & 8.7 \\
\hline & $9 \mathrm{dS} / \mathrm{m}$ & 75.0 & 8.8 & 7.3 \\
\hline & $12 \mathrm{dS} / \mathrm{m}$ & 65.0 & 6.2 & 7.6 \\
\hline & $15 \mathrm{dS} / \mathrm{m}$ & $66.7 b$ & 3.6 & 5.4 \\
\hline Giza 83 & Control (0) & 66.7 & 13.4 & 7.7 \\
\hline & $6 \mathrm{dS} / \mathrm{m}$ & 73.3 & 11.1 & 8.9 \\
\hline & $9 \mathrm{dS} / \mathrm{m}$ & 81.7 & 9 & 7.8 \\
\hline & $12 \mathrm{dS} / \mathrm{m}$ & 78.3 & 5.8 & 6.4 \\
\hline & $15 \mathrm{dS} / \mathrm{m}$ & 61.7 & 4.4 & 4.6 \\
\hline DR. 101 & Control (0) & 91.7 & 10.3 & 9.3 \\
\hline & $6 \mathrm{dS} / \mathrm{m}$ & 95.0 & 9.7 & 9.1 \\
\hline & $9 \mathrm{dS} / \mathrm{m}$ & 91.7 & 7.3 & 9.4 \\
\hline & $12 \mathrm{dS} / \mathrm{m}$ & 91.7 & 5.1 & 6.8 \\
\hline & $15 \mathrm{dS} / \mathrm{m}$ & 90.0 & 4.1 & 6.1 \\
\hline Giza 111 & Control (0) & 78.3 & 10.7 & 9.6 \\
\hline & $6 \mathrm{dS} / \mathrm{m}$ & 78.3 & 11.4 & 9.5 \\
\hline & $9 \mathrm{dS} / \mathrm{m}$ & 66.7 & 8.2 & 8.2 \\
\hline & $12 \mathrm{dS} / \mathrm{m}$ & 75.0 & 4.9 & 7.2 \\
\hline & $15 \mathrm{dS} / \mathrm{m}$ & 68.3 & 4.4 & 5.9 \\
\hline Giza 22 & Control (0) & 53.3 & 8.7 & 6.1 \\
\hline & $6 \mathrm{dS} / \mathrm{m}$ & 48.3 & 8 & 3.8 \\
\hline & $9 \mathrm{dS} / \mathrm{m}$ & 40.0 & 6.5 & 3.5 \\
\hline & $12 \mathrm{dS} / \mathrm{m}$ & 61.7 & 5.4 & 5.2 \\
\hline & $15 \mathrm{dS} / \mathrm{m}$ & 50.0 & 4.2 & 4.3 \\
\hline L.S.D. (0.05) & & 0.15 & 0.15 & 0.15 \\
\hline
\end{tabular}


El-Rodeny, W. M. et al.

Table (3): Effect of salt stress on plant height and root length of some soybean genotypes at 50 days from sowing.

\begin{tabular}{|c|c|c|c|}
\hline \multirow{2}{*}{\multicolumn{2}{|c|}{ Genotypes }} & Plant height/ $(\mathrm{cm})$ & Root length $(\mathrm{cm})$ \\
\hline & & $\begin{array}{c}17.52 \\
212\end{array}$ & 10.42 \\
\hline \multirow{2}{*}{\multicolumn{2}{|c|}{\begin{tabular}{|l|} 
Toano \\
$\mathrm{H} \neg 2 \mathrm{~L} 78$ \\
\end{tabular}}} & $\begin{array}{l}21.2 \\
19.6\end{array}$ & 13.72 \\
\hline & & $\begin{array}{l}19.6 \\
27.77\end{array}$ & 12.58 \\
\hline \multirow{2}{*}{\multicolumn{2}{|c|}{ Giza 35 }} & $\frac{27.77}{29.78}$ & 17.6 \\
\hline & & 29.78 & 20.5 \\
\hline \multicolumn{2}{|l|}{ H32 } & 22.14 & 14.56 \\
\hline \multicolumn{2}{|l|}{ Giza 83} & 17.89 & 9.52 \\
\hline \multicolumn{2}{|l|}{\begin{tabular}{|l|} 
DR. 101 \\
\end{tabular}} & 22.62 & 11.76 \\
\hline \multicolumn{2}{|l|}{ Giza 111} & 20.61 & 11.78 \\
\hline \multicolumn{2}{|l|}{ Giza 22 } & 25.54 & 13.82 \\
\hline \multicolumn{2}{|c|}{ L.S.D. (0.05) } & 6.27 & 0.047 \\
\hline \multirow{2}{*}{\multicolumn{2}{|c|}{$\begin{array}{l}\text { Salinity levels } \\
\text { Control (0) }\end{array}$}} & & \\
\hline & & 24.93 & 15.58 \\
\hline \multicolumn{2}{|c|}{$6 \mathrm{dS} / \mathrm{m}$} & 23.00 & 13.94 \\
\hline $9 \mathrm{dS} / \mathrm{m}$ & & 23.73 & 14.31 \\
\hline $12 \mathrm{dS} / \mathrm{m}$ & & 23.77 & 13.31 \\
\hline $15 \mathrm{dS} / \mathrm{m}$ & & 16.90 & 10.99 \\
\hline L.S.D. (0. & & 4.21 & 0.055 \\
\hline $\mathbf{G}^{*} \mathbf{S}$ & & & \\
\hline H6L136 & Control (0) & 19 & 12.3 \\
\hline & $6 \mathrm{dS} / \mathrm{m}$ & 23.3 & 11.7 \\
\hline & $9 \mathrm{dS} / \mathrm{m}$ & 19.3 & 10 \\
\hline & $12 \mathrm{dS} / \mathrm{m}$ & 13.9 & 9.4 \\
\hline & $15 \mathrm{dS} / \mathrm{m}$ & 12.1 & 8.7 \\
\hline H9L186 & Control (0) & 27.3 & 17.7 \\
\hline & $6 \mathrm{dS} / \mathrm{m}$ & 13.5 & 12.1 \\
\hline & $9 \mathrm{dS} / \mathrm{m}$ & 24 & 15.3 \\
\hline & $12 \mathrm{dS} / \mathrm{m}$ & 21.3 & 12 \\
\hline & $15 \mathrm{dS} / \mathrm{m}$ & 20 & 11.5 \\
\hline Toano & Control (0) & 24.3 & 17 \\
\hline & $6 \mathrm{dS} / \mathrm{m}$ & 22.4 & 15.3 \\
\hline & $9 \mathrm{dS} / \mathrm{m}$ & 21.7 & 12.3 \\
\hline & $12 \mathrm{dS} / \mathrm{m}$ & 15.7 & 9.3 \\
\hline & $15 \mathrm{dS} / \mathrm{m}$ & 14 & 9 \\
\hline $\mathrm{H} \neg 2 \mathrm{~L}$ 78 & Control (0) & 30 & 19 \\
\hline & $6 \mathrm{dS} / \mathrm{m}$ & 25.5 & 18.4 \\
\hline & $9 \mathrm{dS} / \mathrm{m}$ & 19.3 & 16.3 \\
\hline & $12 \mathrm{dS} / \mathrm{m}$ & 16.7 & 18 \\
\hline & $15 \mathrm{dS} / \mathrm{m}$ & 14.2 & 16.3 \\
\hline Giza 35 & Control (0) & 32 & 22 \\
\hline & $6 \mathrm{dS} / \mathrm{m}$ & 31.5 & 20.1 \\
\hline & $9 \mathrm{dS} / \mathrm{m}$ & 30 & 20 \\
\hline & $12 \mathrm{dS} / \mathrm{m}$ & 29.9 & 21 \\
\hline & $15 \mathrm{dS} / \mathrm{m}$ & 25.6 & 19.4 \\
\hline H32 & Control (0) & 19 & 10 \\
\hline & $6 \mathrm{dS} / \mathrm{m}$ & 21.7 & 14.8 \\
\hline & $9 \mathrm{dS} / \mathrm{m}$ & 25 & 15.3 \\
\hline & $12 \mathrm{dS} / \mathrm{m}$ & 28.7 & 20.3 \\
\hline & $15 \mathrm{dS} / \mathrm{m}$ & 16.3 & 12.5 \\
\hline Giza 83 & Control (0) & 22 & 12.7 \\
\hline & $6 \mathrm{dS} / \mathrm{m}$ & 21.4 & 11.4 \\
\hline & $9 \mathrm{dS} / \mathrm{m}$ & 23 & 12.7 \\
\hline & $12 \mathrm{dS} / \mathrm{m}$ & 12 & 5.6 \\
\hline & $15 \mathrm{dS} / \mathrm{m}$ & 10.4 & 5.2 \\
\hline DR. 101 & Control (0) & 27.7 & 14.7 \\
\hline & $6 \mathrm{dS} / \mathrm{m}$ & 20.3 & 9.3 \\
\hline & $9 \mathrm{dS} / \mathrm{m}$ & 22.7 & 12.5 \\
\hline & $12 \mathrm{dS} / \mathrm{m}$ & 23 & 14 \\
\hline & $15 \mathrm{dS} / \mathrm{m}$ & 19.4 & 8.3 \\
\hline Giza 111 & Control (0) & 21.7 & 13.7 \\
\hline & $6 \mathrm{dS} / \mathrm{m}$ & 23.4 & 11.5 \\
\hline & $9 \mathrm{dS} / \mathrm{m}$ & 24.3 & 15.7 \\
\hline & $12 \mathrm{dS} / \mathrm{m}$ & 18 & 10 \\
\hline & $15 \mathrm{dS} / \mathrm{m}$ & 15.6 & 8 \\
\hline Giza 22 & Control (0) & 26.3 & 16.7 \\
\hline & $6 \mathrm{dS} / \mathrm{m}$ & 27.3 & 14.9 \\
\hline & $9 \mathrm{dS} / \mathrm{m}$ & 28 & 13 \\
\hline & $12 \mathrm{dS} / \mathrm{m}$ & 24.6 & 13.5 \\
\hline & $15 \mathrm{dS} / \mathrm{m}$ & 21.5 & 11 \\
\hline L.S.D. (0. & & 13.261 & 0.2209 \\
\hline
\end{tabular}


Table (4): Effect of salt stress on ion percentage of soybean tissue

\begin{tabular}{|c|c|c|c|c|c|}
\hline G & notypes & Shoot K+ (\%) & Root K+ (\%) & shoot $\mathrm{Na}+(\%)$ & Root Na+ (\%) \\
\hline H6L136 & & 5.98 & 2.8 & 2.24 & 5.56 \\
\hline H9L186 & & 4.02 & 2.88 & 6.86 & 3.24 \\
\hline Toano & & 4.48 & 2.12 & 1.6867 & 3.72 \\
\hline $\mathrm{H} \neg 2 \mathrm{~L} 78$ & & 7.2 & 2.78 & 6.8 & 7.46 \\
\hline Giza 35 & & 4.54 & 2 & 2.16 & 3.88 \\
\hline H32 & & 7.25 & 2.86 & 5.44 & 5.28 \\
\hline Giza 83 & & 7.24 & 2.00 & 2.22 & 4.78 \\
\hline DR. 101 & & 4.58 & 2.00 & 6.94 & 5.30 \\
\hline Giza 111 & & 7.20 & 2.80 & 6.06 & 4.48 \\
\hline Giza 22 & & 5.98 & 2.80 & 6.28 & 3.71 \\
\hline L.S.D. (0. & & 0.09 & 0.07 & 0.10 & 0.07 \\
\hline Salinity I & els & & & & \\
\hline Control & & & & & \\
\hline $6 \mathrm{dS} / \mathrm{m}$ & & 7.85 & 3.48 & 2.86 & 3.59 \\
\hline $9 \mathrm{dS} / \mathrm{m}$ & & 5.68 & 2.38 & 4.06 & 4.05 \\
\hline $12 \mathrm{dS} / \mathrm{m}$ & & 4.40 & 1.15 & 6.50 & 5.59 \\
\hline $15 \mathrm{dS} / \mathrm{m}$ & & 2.55 & 1.41 & 7.54 & 6.56 \\
\hline L.S.D. (0. & & 0.07 & 0.05 & 0.05 & 0.06 \\
\hline$G * S$ & & & & & \\
\hline H6L136 & Control (0) & 9.80 & 4.60 & 0.90 & 4.20 \\
\hline & $6 \mathrm{dS} / \mathrm{m}$ & 8.40 & 3.80 & 1.10 & 3.50 \\
\hline 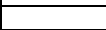 & $9 \mathrm{dS} / \mathrm{m}$ & 5.20 & 3.40 & 2.10 & 4.10 \\
\hline & $12 \mathrm{dS} / \mathrm{m}$ & 4.10 & 1.60 & 3.40 & 6.30 \\
\hline & $15 \mathrm{dS} / \mathrm{m}$ & 2.40 & 0.60 & 3.70 & 9.80 \\
\hline H9L186 & Control (0) & 6.10 & 3.90 & 3.50 & 2.80 \\
\hline & $6 \mathrm{dS} / \mathrm{m}$ & 5.30 & 3.30 & 4.10 & 2.40 \\
\hline & $9 \mathrm{dS} / \mathrm{m}$ & 3.60 & 1.80 & 6.30 & 2.90 \\
\hline & $12 \mathrm{dS} / \mathrm{m}$ & 3.20 & 0.90 & 9.40 & 3.60 \\
\hline & $15 \mathrm{dS} / \mathrm{m}$ & 1.90 & 4.50 & 10.80 & 4.70 \\
\hline Toano & Control (0) & 6.10 & 3.90 & 0.80 & 5.10 \\
\hline & $6 \mathrm{dS} / \mathrm{m}$ & 5.70 & 3.30 & 1.00 & 2.40 \\
\hline & $9 \mathrm{dS} / \mathrm{m}$ & 4.40 & 1.80 & 1.20 & 2.90 \\
\hline & $12 \mathrm{dS} / \mathrm{m}$ & 3.90 & 0.90 & 2.20 & 3.60 \\
\hline & $15 \mathrm{dS} / \mathrm{m}$ & 2.30 & 0.70 & 3.20 & 4.70 \\
\hline $\mathrm{H} \neg 2 L 78$ & Control (0) & 10.80 & 4.40 & 3.50 & 5.10 \\
\hline & $6 \mathrm{dS} / \mathrm{m}$ & 9.80 & 3.90 & 4.10 & 8.30 \\
\hline & $9 \mathrm{dS} / \mathrm{m}$ & 7.40 & 3.20 & 6.30 & 9.80 \\
\hline & $12 \mathrm{dS} / \mathrm{m}$ & 5.10 & 1.70 & 9.40 & 10.20 \\
\hline & $15 \mathrm{dS} / \mathrm{m}$ & 2.90 & 0.60 & 10.80 & 3.90 \\
\hline Giza 35 & Control (0) & 6.40 & 3.70 & 0.70 & 4.80 \\
\hline & $6 \mathrm{dS} / \mathrm{m}$ & 5.90 & 3.20 & 1.20 & 2.30 \\
\hline & $9 \mathrm{dS} / \mathrm{m}$ & 4.20 & 1.60 & 1.90 & 3.00 \\
\hline & $12 \mathrm{dS} / \mathrm{m}$ & 3.70 & 0.80 & 3.10 & 3.70 \\
\hline & $15 \mathrm{dS} / \mathrm{m}$ & 2.50 & 0.70 & 3.70 & 5.60 \\
\hline H32 & Control (0) & 10.40 & 4.70 & 3.20 & 5.00 \\
\hline & $6 \mathrm{dS} / \mathrm{m}$ & 9.60 & 3.90 & 3.90 & 4.20 \\
\hline & $9 \mathrm{dS} / \mathrm{m}$ & 7.50 & 3.50 & 4.80 & 3.00 \\
\hline & $12 \mathrm{dS} / \mathrm{m}$ & 5.70 & 1.50 & 6.90 & 6.90 \\
\hline & $15 \mathrm{dS} / \mathrm{m}$ & 3.40 & 0.70 & 8.30 & 7.40 \\
\hline Giza 83 & Control (0) & 10.90 & 3.70 & 0.90 & 2.30 \\
\hline & $6 \mathrm{dS} / \mathrm{m}$ & 9.90 & 3.20 & 1.10 & 3.00 \\
\hline & $9 \mathrm{dS} / \mathrm{m}$ & 7.50 & 1.60 & 2.10 & 3.70 \\
\hline & $12 \mathrm{dS} / \mathrm{m}$ & 5.20 & 0.80 & 3.40 & 5.60 \\
\hline & $15 \mathrm{dS} / \mathrm{m}$ & 2.70 & 0.70 & 3.70 & 9.30 \\
\hline DR. 101 & Control $(0)$ & 6.50 & 3.70 & 3.40 & 4.80 \\
\hline & $6 \mathrm{dS} / \mathrm{m}$ & 5.70 & 3.20 & 4.30 & 4.10 \\
\hline & $9 \mathrm{dS} / \mathrm{m}$ & 4.40 & 1.60 & 6.70 & 3.70 \\
\hline & $12 \mathrm{dS} / \mathrm{m}$ & 3.90 & 0.80 & 9.50 & 5.60 \\
\hline & $15 \mathrm{dS} / \mathrm{m}$ & 2.40 & 0.70 & 10.80 & 8.30 \\
\hline Giza 111 & Control (0) & 10.80 & 4.60 & 3.00 & 2.60 \\
\hline & $6 \mathrm{dS} / \mathrm{m}$ & 9.80 & 3.80 & 3.70 & 3.00 \\
\hline & $9 \mathrm{dS} / \mathrm{m}$ & 7.40 & 3.40 & 5.60 & 4.20 \\
\hline & $12 \mathrm{dS} / \mathrm{m}$ & 5.10 & 1.60 & 8.30 & 5.70 \\
\hline & $15 \mathrm{dS} / \mathrm{m}$ & 2.90 & 0.60 & 9.80 & 6.90 \\
\hline Giza 22 & Control (0) & 9.80 & 3.80 & 3.50 & 2.40 \\
\hline & $6 \mathrm{dS} / \mathrm{m}$ & 8.40 & 3.20 & 4.10 & 2.90 \\
\hline & $9 \mathrm{dS} / \mathrm{m}$ & 5.20 & 1.80 & 6.30 & 3.60 \\
\hline & $12 \mathrm{dS} / \mathrm{m}$ & 4.10 & 0.90 & 9.40 & 4.70 \\
\hline & $15 \mathrm{dS} / \mathrm{m}$ & 2.40 & 4.30 & 10.80 & 5.10 \\
\hline L.S.D. (0. & & 0.22 & 0.16 & 0.18 & 0.18 \\
\hline
\end{tabular}

According to the results, it can be concluded that genotypes Toano, Giza 83 and DR. 101 are salt stress tolerant due to its low $\mathrm{Na}+$ absorption and high $\mathrm{Na}+$ accumulation in root compared with the other studied genotypes. 
Additionally, the highest $\mathrm{K}+$ absorption by genotypes Toano, Giza 83 and DR. 101 may be the main factor of to salt stress tolerant during germination and seedling establishment stages in comparison to the other studied genotypes. These results have conformity with the Chippa and Rana, 1995. There is a relationship between potassium decrease and sodium increase in seedling tissue with the sensitivity to salinity. Sodium affected the cell membrane permeability, deconstructed the cell membrane and destroyed the selectivity property. Other investigations demonstrated that resistant plants to salinity not only have the lower sodium - potassium ratio in compareing with sensitive plants to salinity, but also deposited high sodium in root tissue , therefore it inhibits the sodium transmit to shoot tissue, also inhibits their damage. The results of this study showed that genotypes Toano, Giza 83 and DR. 101 were the highest tolerance to salinity among all studied genotypes.

In conclusion, according to the results, it could be concluded that cultivars Toano, Giza 83 and DR. 101 are salt stress tolerant due to its low $\mathrm{Na}+$ absorption and high $\mathrm{Na}+$ accumulation in root compared with the other studied genotypes. The results indicated that under salt stress plant growth of cultivars Toano, Giza 83 and DR. 101 were better compared to the other soybean cultivars.

\section{REFERENCES}

Ansari, R. and T.J. Flowers, (1986). Leaf to leaf distribution of ions in some monocotyledonous plants grown under saline conditions. In Prospects of Biosaline Research. Eds. R. Ahmed and A. San Pietro. University of Karachi, Karachi, pp: 167-181.

Chen, D.; D.M. Yu-Renpei and R.P. Yu, (1996). Studies of relative salt tolerance of crops. Salt tolerance of some main crop species. Actapedologica science, 33: 121-128.

Chippa B.R. and D. Rana, (1995). $\mathrm{Na}+/ \mathrm{K}+$ ratio as the basis of salt tolerance in wheat. Australian Journal of Agriculture Research, 46: 533539.

Farhoudi R.; and M. Tafti (2011) Effect of Salt Stress on Seedlings Growth and lons Homeostasis of Soybean (Glysin max L.) Cultivars Advances in Environmental Biology, 5(8): 2522-2526

Farhoudi, R.; F. Sharifzadeh; M. Makkizadeh and M., Kochakpour, (2007). The effects of $\mathrm{NaCl}$ priming on salt tolerance in canola (Brassica napus) seedlings grown under

Jalali M. H. Merikhpour; M. J. Kaledhonkar and S.E.A.T.M. Van Der Zee (2008). Effects of waste water irrigation on soil sodicity and nutrient leaching in calcareous soils. Agricultural water management vol., 95 Issue (2) PP. 143-153.

Khajeh-Hosseini, M.; A.A. Powell and I.J. Bingham, (2003).The interaction between salinity stress and seed vigour during germination of soybean seeds. Seed Science and Technology, 31: 715-725

Okçu, G., M.D. Kaya and M. Atak, (2005). Effects of salt and drought stresses on germination and seedling growth of pea (Pisum sativumL.). Turk. J. Agric. For, 29(4): 237-242.

Sadeghi, H., (2009). Effects of Different Levels of Sodium Chloride on Yield and Chemical Composition in Two Barley Cultivars, Am.-Eurasian J. Sustain. Agric., 3(3): 314-320. 
Rahimi, A and A. Biglarifard, (2011). Impacts of $\mathrm{NaCl}$ Stress on Proline, Soluble Sugars, Photosynthetic Pigments and Chlorophyll Florescence of Strawberry. Advances in Environmental Biology, 5(4): 617-623

Rehman, S., P.J.C. Harris, W.F. Bourne and J. Wilkin, (1996). The effect of sodium chloride on germination and the potassium and calcium content of Acacis seeds. Seed Sci. and Technol., 25: 45-57

Rogress, M.E., C.L. Noble, G.M. Halloran and M.E. Nicolas. (1994) The effect of $\mathrm{NaCl}$ on the germination and early seedling growth of white clover (Trifolium repens) population selected for high and low tolerance. Seed science and technology, 23: 277-287.

Wang, D. and M.C.,Shannon, (1999). Emergence and seedling growth of soybean cultivars and maturity groups under salinity. Plant Soil, 214: 117-124.

Zayed B.A. (2002). Performance of some rice cultivars as affected by irrigation and potassium fertilizer treatment under saline soil conditions. PhD thesis, agron. Dept. Fac. of Agric. Mansoura Univ., Egypt.

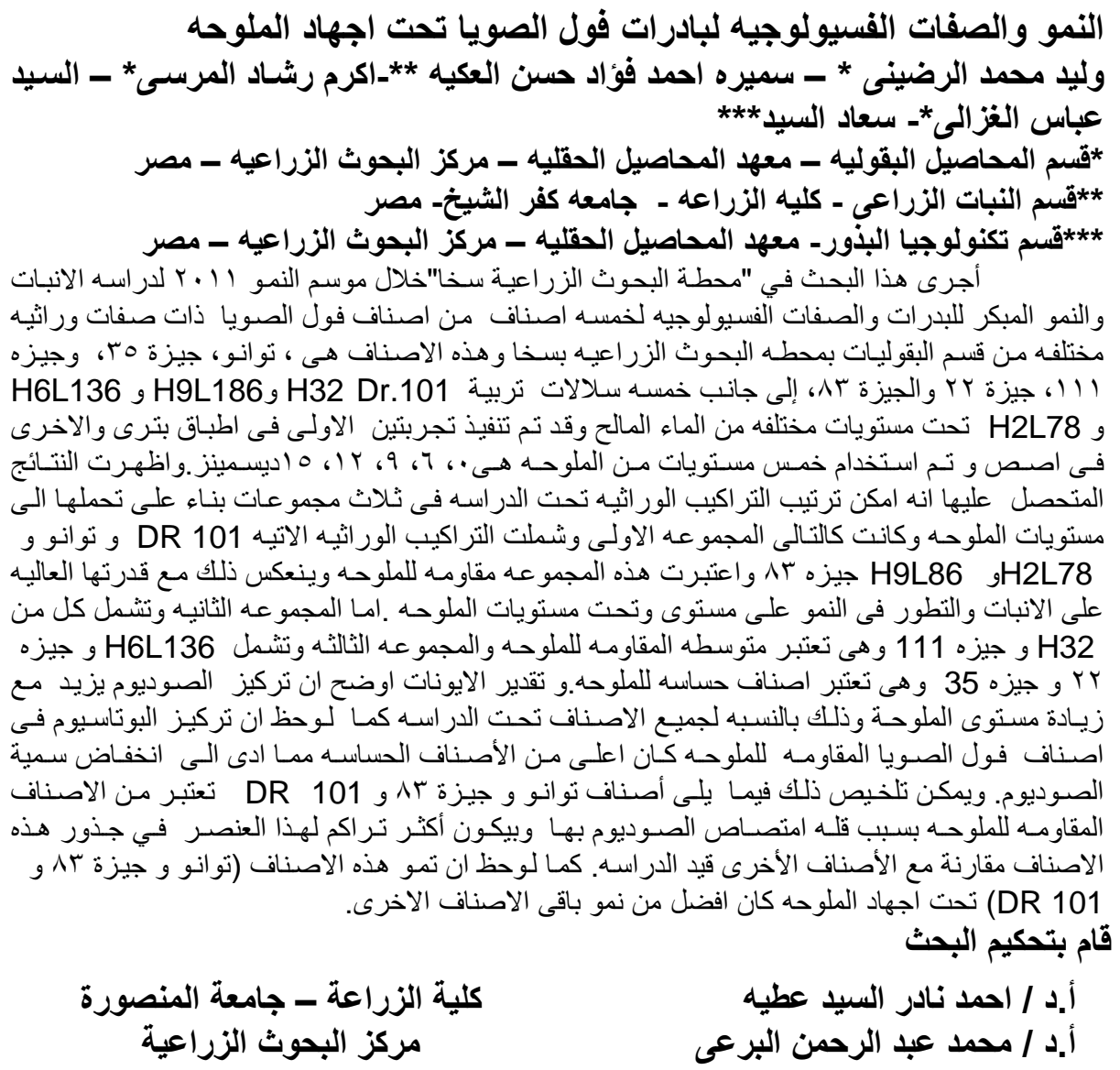

